\title{
Integrated Photodiodes in Standard CMOS Technology for CD and DVD Applications
}

\author{
Saša Radovanović, AnneJohan Annema and Bram Nauta
}

\begin{abstract}
The influence of two different geometries (layouts) and two structures of high-speed photodiodes in fully standard $0.18 \mu \mathrm{m}$ CMOS technology on their intrinsic (physical) and electrical bandwidths is analyzed. In addition, a possible application of the integrated photodiodes for the CD and DVD optical pick-up units is discussed. Two photodiode structures with a highest responsivity are studied: nwell $/ \mathrm{p}$ substrate and $\mathrm{p}+/$ nwell/p-substrate (double photodiode). The photodiode bandwidths are compared for $\lambda=780 \mathrm{~nm}$ and $\lambda=650 \mathrm{~nm}$ wavelength, corresponding to the lasers for CD and DVD, respectively. Slow substrate current component limits the intrinsic bandwidth of nwell/p-substrate and $\mathrm{p}+/$ nwell/p-substrate photodiodes to $6 \mathrm{MHz}$ and $7 \mathrm{MHz}$, for a CD application as well as $70 \mathrm{MHz}$ and $100 \mathrm{MHz}$ for a DVD application. The electrical bandwidth of these diodes in combination with typical transimpedance amplifiers, will be always larger than the calculated intrinsic bandwidths meaning that the diode capacitance is not critical in total phptoreceiver design.
\end{abstract}

\section{INTRODUCTION}

The motivation to produce high-speed optical detectors in fully standard CMOS technology has increased markedly as the electronic speed of today's CMOS circuits is well in $\mathrm{GHz}$ range. In order to keep the cost of the short-haul data system low, monolithically integrated CMOS optical detectors are preferred in both data communication [1] and optical storage systems (inside CD/DVD pick-up units) [2]. The integrated photo diodes avoid the relatively high capacitance of discrete photo diodes (device and interconnect) and its influence on the bandwidth.

In the literature, several methods have been implemented to obtain high-frequency photodetectors in standard CMOS. In [3], the spatially modulated light cletector, which cancels the effect of the slow substrate carriers, is introduced. As a tradeoff, the responsivity of the photodiode is sacrificed. A high-speed finger $n+/ p-$ substrate CMOS photodiode for optical storage system is presented in [5]; here, the photodiode performance for four different geometries and one diode structure is discussed based on only time-response measurements.

In this paper the influence of various structures and geometries (layouts) of CMOS photodiodes on their bandwiclth is analytically analyzed. The analyzed

Sașa Radovanović, AnneJohan Annema and Bram Nauta are with the Department of Integrated Circuit Design, University of Twente, MESA + institute,P.O. Box 217, Enschede, 7500 AE, The Netherlands, E-mail: s.radovanovic@utwente.n! diodes have the same area; it provides easier comparison of their responsivity. The bandwidths are compared for $\lambda=780 \mathrm{~nm}$ and $\lambda=650 \mathrm{~nm}$ wavelength lights which are used in today's CD and DVD optical pick-up units. The physical and electrical bandwidth characteristics for nwell/p-substrate; $\mathrm{p}+/$ nwell/p-substrate diode are discussed. The total bandwidth is by approximation the lower of these two.

\section{INTRINSIC (PHYSICAL) PHOTODIODE BANDWIDTH}

\section{A. Finger nwell/psubstrate photodiode}

General intrinsic frequency behavior of standard CMOS photodiodes is investigated on a minimaldistance finger nwell/p-substrate diode, shown in Fig 1. Photodiode responses for both input wavelengths $\lambda=780 \mathrm{~nm}$ and $\lambda=650 \mathrm{~nm}$ are calculated with the following procedure:

The overall photodiode response is the sum of the drift and the diffusion photocurrents inside the diode volume. Firstly, the frequency response of the nwell diffusion current under impulse light radiation is solved analytically in two-dimensions following a procedure similar to that in [3]. The surface is assumed to be reflective (the normal component of the gradient of the carrier density is zero) and the electron densities on the other three nwell sides are assumed to be zero.

Secondly, we solved the substrate current response using an one-dimensional (vertical) diffusion equation. The two "p" layers are placed at the top of each other (Figure 1). There is a boundary condition between the two layers related to both the current density and the minority carrier concentration. Due to the continuity of currents, the current densities are equal in a plane between the two layers:

$$
-\left.q D_{n 1} \frac{\partial n_{1}(x, s)}{\partial x}\right|_{x=L_{e p i}}=-\left.q D_{n 2} \frac{\partial n_{2}(x, s)}{\partial x}\right|_{x=L_{i p ; i}}
$$

where $D_{n 1}, D_{n 2}$ represent the diffusion coefficients and $n_{1}$ and $n_{2}$ are the density of the minority carriers in the epitaxial layer and the substrate, respectively. The second boundary condition is related to the continuity of the concentration of the minority carriers:

$$
n_{1}\left(L_{e p i}, s\right)=n_{2}\left(L_{e p i}, s\right)
$$

where $L_{e p i}$ is the depth between the bottom of the depletion region and the bottom of the p-epi substrate. 
The other two boundary conditions for both electron densities at the bottom of the depletion region and at the infinite substrate depth are taken to be zero. The infinitely large substrate is taken in order to avoid long and complex calculations. Finite substrate depth would certainly result in a faster diode response [8]. The third addend, the drift current responses are taken independent of frequency for simplicity reasons since their bandwidth is much larger than the bandwidth of diffusion currents. The amount of the drift current is directly related to the depletion volume in which carriers are generated.

\section{A.1 CD application}

Generally, for $\lambda=780 \mathrm{~nm}$ light, the substrate current dominates the overall photocurrent frequency behavior up to few hounders of $\mathrm{MHz}$, also illustrated in Fig 2. Since the cuttof frequency of the substrate current is in the range of only a few $\mathrm{MHz}$, this significantly limits the overall photodiode bandwidth. Both the fast diffusion response in nwells and the fast drift current response are overshadowed with this large substrate current (Fig 2). A diode geometry has no influence on a total diode bandwidth. Maximal data-rate (for CD application) with non-return-to-zero data is about $18 \mathrm{Mb} / \mathrm{s}$ which is about $13 \mathrm{x}$ CD speed. In order to increase the photodiode bandwidth, the designers has to minimize the effect of the slow substrate current.

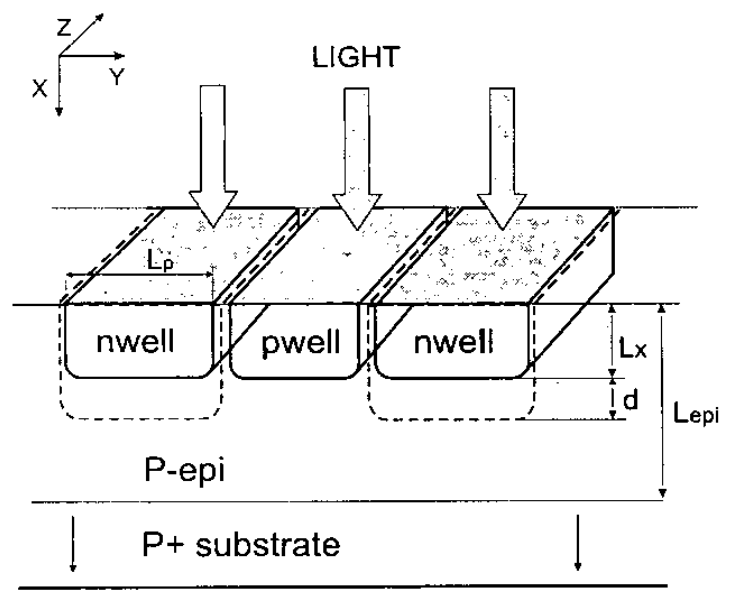

Fig. 1. Finger nwell/p-substrate photodiode structure in standard CMOS technology

\section{A.2 DVD application}

Fig 3. shows the calculated intrinsic (physical) frequency responses of the two finger nwell/p-substrate diodes with $2 \mu \mathrm{m}$ and $10 \mu \mathrm{m}$ nwell sizes, for $\lambda=650$ $\mathrm{nm}$ wavelength. The values for the parameters in the analytical expressions were directly obtained from the

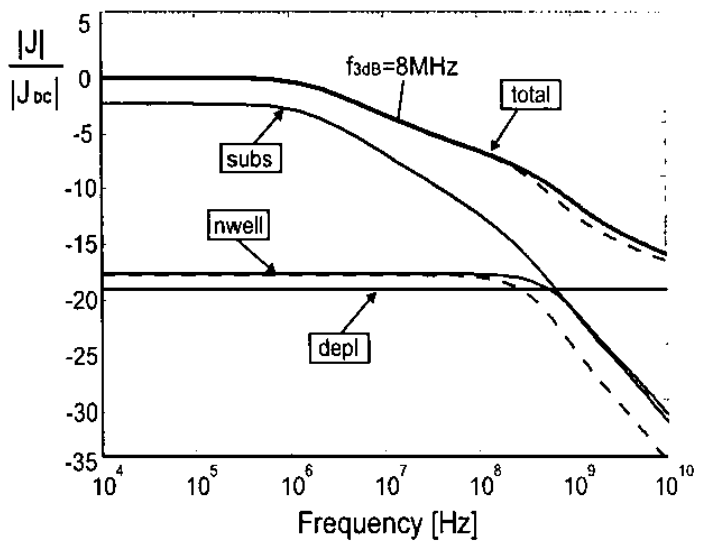

Fig. 2. Calculated amplitude response of nwell/p-substrate photodiodes with: $2 \mu \mathrm{m}$ (solid lines) and $10 \mu$ nwell size (dashed lines) for $\lambda=780 \mathrm{~nm}$

process technology parameters for a fully standard 0.18 $\mu \mathrm{m}$ CMOS process.

A slow substrate current dominates the overall diode response. Generated holes need time to diffuse towards the junctions above and limit the substrate current bandwidth to $28 \mathrm{MHz}$. The larger bandwidtl of the nwell diffusion current is mainly determined by the length of the shortest side of the nwell, which further determines the charge gradient responsible for the diffusion process. For $L_{p}=2 \mu \mathrm{m}$ the shortest sides are both lateral and vertical dimensions. The charge gradient is high and $f_{3 d B n w e l l}=2 \mathrm{GHz}$. For $L_{p}=10 \mu \mathrm{m}$, the shortest side is the nwell depth and the charge gradient is lower than in the previous case, $f_{3 d B_{n} \text { well }}=1 \mathrm{GHz}$. Thus, the larger the nwell width $L_{p}$, in comparison with its depth $L_{x}\left(L_{p}>2 L_{x}\right)$, the lower its infiuence on the hole diffusion current bandwidth (see Fig 3.). The overall maximal intrinsic photodiode bandwidth is $160 \mathrm{MHz}$ and the photodiode geometry has again almost no influence on it. The slow response decay after a $-3 \mathrm{~dB}$ point is due to the combination of three current components in the diode. Maximal data-rate (for DVD application) with non-return-to-zero data is about $360 \mathrm{Mb} / \mathrm{s}$ which is about 10x DVD speed.

\section{B. Finger $p+/ n w e l l / p$-substrate photodiode}

The second diode structure analytically analyzed in this paper is a finger $\mathrm{p}+/$ nwell/ $\mathrm{p}$-substrate structure (Fig 4). The diffusion current responses are again calculated using a two-dimensional diffusion equation, given in [3]. Main difference in comparison with nwell/psubstrate photodiode analyzed in previous section is diffusion response inside nwell region. The boundary conditions for the holes density on every nwell side are zero since it is enclosed by junctions:

$$
\left.p_{n}\right|_{x, y @ \text { boundary }}=0
$$




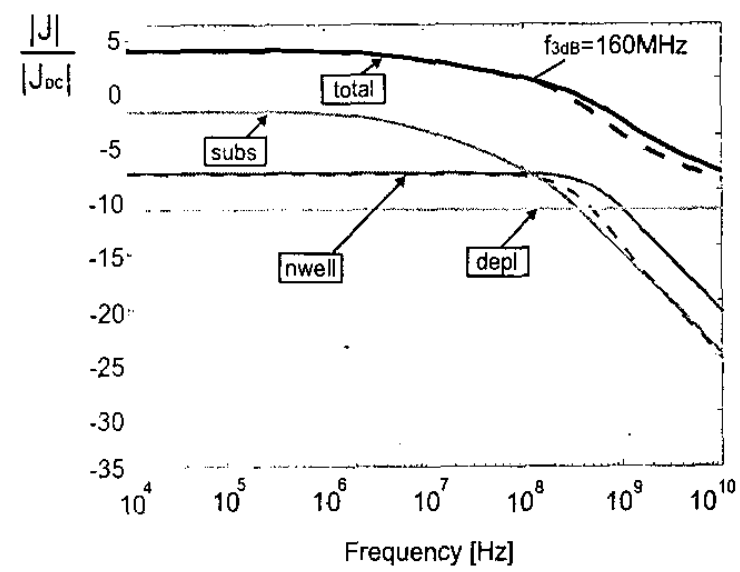

Fig. 3. Calculated amplitude response of nwell/p-substrate photodiodes with: $2 \mu \mathrm{m}$ (solid lines) and $10 \mu$ nwell size (dashed lines) for $\lambda=650 \mathrm{~nm}$

The current response of the nwell is calculated and final result is given in eq. 1. in [8]. For the p+region, the hole current response is calculated using the nwell response in previously analyzed diode and changing diffusion coefficient and diffusion length as well the depth of the junction $\left(D_{p 1} \rightarrow D_{n 1}, L_{p 1} \rightarrow L_{n 1}, L_{x} \rightarrow L_{x 1}\right)$. The substrate current response for both diodes is the same due to the same nwell depths.

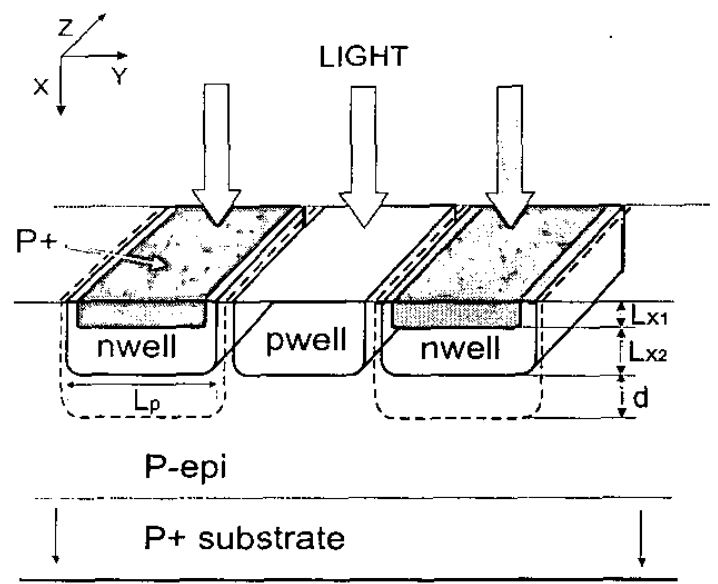

Fig. 4. Finger $\mathrm{p}+/$ nwell/p-substrate photodiode structure in standard CMOS technology with $\mathrm{p}+/ \mathrm{p}$-epi substrate in a twin-well.

\section{B.1 CD application}

The frequency responses of two finger $\mathrm{p}+/$ nwell $/ \mathrm{p}$ substrate diodes with $2 \mu \mathrm{m}$ and $10 \mu \mathrm{m}$ nwell sizes, for $\lambda=8.50 \mathrm{~nm}$ wavelength are shown in Fig 5 . This picture illustrates that the frequency bandwidths of $p+$ and nwell currents are mainly determined by the low physical depth of the junctions $\left(2 L_{x 1}<L_{p}\right)$; changing nwell and $\mathrm{p}+$ widths has almost no effect on the cutoff frequency. The bandwidth of the junction-framed nwell current is $f_{3 d B \text { nwell }}=5 \mathrm{GHz}$ for $L_{p}=2 \mu \mathrm{m}$, and $f_{3 d B n w e l l}=4.2 \mathrm{GHz}$ for $L_{p}=10 \mu \mathrm{m}$. That is more than twice the nwell bandwidth of the nwell/p-substrate diode. The distances towards the junctions are lower providing higher charge gradient and faster diffusion process. The current bandwidth of the $\mathrm{p}+$ region is lower than the bandwidth of the nwell current; the calculated value is about $3 \mathrm{GHz}$ for all diode geometries. The p+ surface is reflective for the carriers that are repelled back to the other three $\mathrm{p}+$ sides with the junctions. Thus, the carriers need extra time to start contributing to the overall photocurrent. The intrinsic photodiode bandwidth is $8 \mathrm{MHz}$, and the photodiode geometry has no influence on it (see Figure 4). Maximal data-rate (for $\mathrm{CD}$ application) with non-return-to-zero data is again about $13 \mathrm{x}$ CD speed.

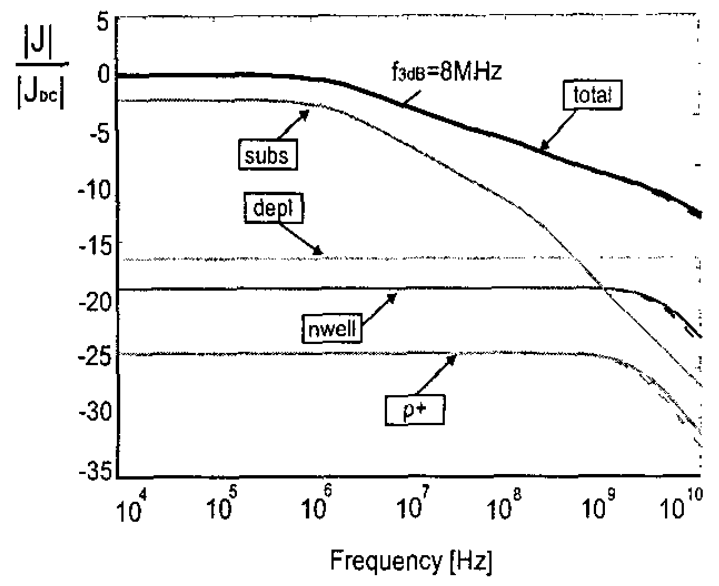

Fig. 5. Calculated amplitude response of $p+/$ nuell $/ p$-substrate photodiode with $\mathrm{p}+/ \mathrm{p}$-epi substrate in a twin-well technology: $2 \mu \mathrm{m}$ (solid lines) and $10 \mu \mathrm{m}$ nwell size (dashed lines) for $\lambda=780 \mathrm{~nm}$

\section{B.2 DVD application}

The frequency responses of two finger $\mathrm{p}+/$ nwell $/ \mathrm{p}$ substrate diodes with $2 \mu \mathrm{m}$ and $10 \mu \mathrm{m}$ nwell sizes, for $\lambda=650 \mathrm{~nm}$ wavelength are shown in Fig 6 . The bandwidth of the junction-framed nwell current is $f_{3 d B n w e l l}=5 \mathrm{GHz}$ for $L_{p}=2 \mu \mathrm{m}$, and $f_{3 d B n w e l l}=4 \mathrm{GHz}$ for $L_{p}=10 \mu \mathrm{m}$. That is more than twice the nwell bandwidth of the nwell/psubs diode because of the lower distances towards junctions and thus, higher charge gradient leading to a faster diffusion process. The current bandwidth of the $\mathrm{p}+$ region is lower than the nwell current and is about $3 \mathrm{GHz}$ for all diode geometries. This is expected, since its surface is reflective for the carriers that are repelled back towards other three $\mathrm{p}+$ sides 
with junctions; this is an extra time they need to start contributing to the overall photocurrent.

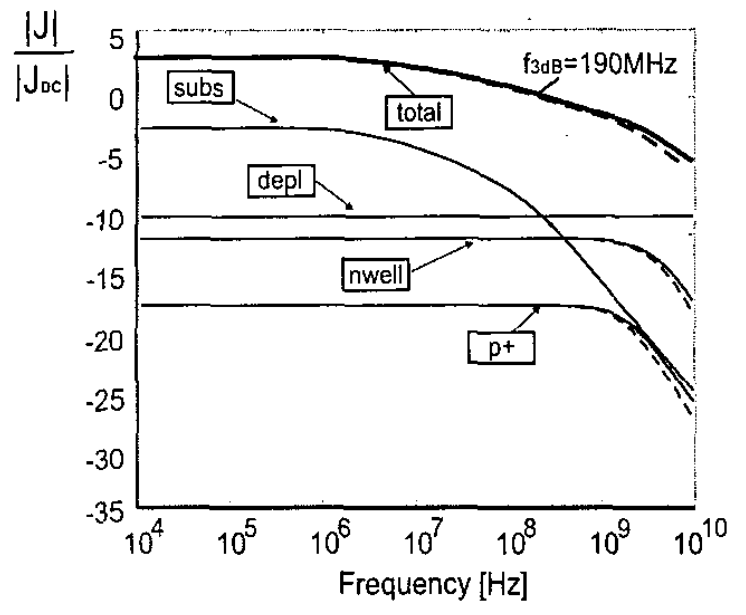

Fig. 6. Calculated amplitude response of $p+/ n w e l l / p$-substrate photodiode with $\mathrm{p}+/ \mathrm{p}$-epi substrate in a twin-well technology: $2 \mu \mathrm{m}$ (solid lines) and $10 \mu \mathrm{m}$ nwell size (dashed lines) for $\lambda=650 \mathrm{imm}$

The overall intrinsic photodiode bandwidth is 190 $\mathrm{MHz}$, and the photodiode geometry has no influence on it (see Fig. 6). Maximal data-rate (for DVD application) with non-return-to-zero data is $400 \mathrm{Mb} / \mathrm{s}$ which is about 16x DVD speed.

\section{EXTRINSIC (ELECTRICAL) PHOTODIODE BANDWIDTH}

Apart from the intrinsic bandwidth, the in-circuit photodiode bandwidth is also determined by the extrinsic (electrical) bandwidth, which is proportional to the diode parasitic capacitance. Table 1 . shows calculated values of the parasitic capacitances for two different geometries of nwell/p-substrate and p+/nwell/psubstrate photodiodes. The depletion region depths that strongly determine diode capacitance are calculated from the process technology parameters. For all discussed finger photodiodes, by decreasing the diode nwell size (for a constant diode area), the parasitic capacitance increases. In order to keep the total bandwidth high (for $\mathrm{pF}$ diode capacitance), the subsequent transimpedance amplifier (TIA) should be designed to have low input impedance $(\leq 50 \Omega)$. Two possible solutions using common source and common gate amplifiers presented in [8].

\section{CONCLUSION}

We presented integrated photodiodes with two structures and two different geometries in standard $0.18 \mu \mathrm{m}$ CMOS technology that can be used for a CD and a DVD optical pick-up units. Maximal speed for CD unit for
TABLE I

PARASITIC CAPACITANCE FOR DIFFERENT PHOTODIODE STRUCTURES

\begin{tabular}{|c|c|c|}
\hline \hline diode structure & nwell size & capacitance \\
\hline nwell/psubstrate & $2 \mu \mathrm{m}$ & $2.42 \mathrm{pF}$ \\
\hline nwell/psubstrate & $10 \mu \mathrm{m}$ & $0.63 \mathrm{pF}$ \\
\hline $\mathrm{p}+/ \mathrm{nwell} /$ psubstrate & $2 \mu \mathrm{m}$ & $4.63 \mathrm{pF}$ \\
\hline $\mathrm{p}+/ \mathrm{nwell} /$ psubstrate & $.10 \mu \mathrm{m}$ & $2.83 \mathrm{pF}$ \\
\hline
\end{tabular}

both nwell/p-substrate and $\mathrm{p}+/$ nwell/p-substrate photodiode is about $13 \mathrm{x} C D$ speed. In order to increase this speed the effect of slow substrate carriers has to be minimized.

For DVD applications on the other hand, the speed of analyzed photodiodes is 13x DVD (nwell/p-substrate) and 16X DVD ( $\mathrm{p}+/$ nwell/p-substrate) and that corresponds to state-of-the-art achievable data-rates while the cost of the total pick-up unit can be minimized.

The diode geometries have almost no influence on their overall intrinsic bandwidth. The electrical photodiode bandwidth determined by the parasitic diode capacitances and the input impedance of the typical TIA's $(\leq 50 \Omega)$, will be in the $\mathrm{GHz}$ range and will not limit the total diode bandwidth.

For deeper nwells (related to the technology), and for lower wavelengths $(\lambda<650 \mathrm{~nm})$, photodiode geometry will certainly become important because of the smaller contribution of slow substrate current.

\section{REFERENCES}

[1] I Radovanović, W. van Etten and H. Freriks: "Ethernet Based Passive Optical Local Area Networks for Fiber-tothe-Desk Application", to be published in Journal of Lightwave Technology, the Special Issue on optical networks in November 2003.

[2] Gerben W. de Jong, Jozef R.M. Bergervoet, Johannes H.A. Brekelmans, Job F.P. van Mill:" A DC-to- $250 \mathrm{MHz}$ Current Pre-Amplifier with Integrated Photo-Diodes in Standard CBiMOS, for Optical-Storage Systems", ISSCC 2002, 6. February, USA.

[3] J. Genoe, D. Coppée, J. H. Stiens, R. A. Vounckx and M. Kuijk: 'Calculation of the current response of the spatially' modulated light CMOS detector', IEEE Trans. Electr. Devices, vol. 48 , No. 9, pp. 1892-1902, 2001.

44] S. M. Sze: 'Physics of semiconductor devices', New York: Wiley-Interscience, 2-1nd edition, p. 81, 1981.

[5] H. Zimmermann, H. Dietrich A. Ghazi, P. Seegebrecht: "Fast CMOS-Integrated Finger Photodiodes for a Wide Spectral Range", ESSDERC 2002, pp. 435-438, 24-25 September, Italy.

[6] C.H. Lu and W.Z. Chen: "Bandwidth enhancement techniques for transimpedance amplifier in CMOS techniques": ESSCIRC 2001, Villach, Austria, 18-20 September 2001.

[7] C. Tomazu and S.M. park: "Wideband low-noise CMOS transimpedance amplifier for gigahertz operation", Electronic Letters, pp. 1194-1196, vol. 32, June 1996.

[8] S. Radovanović, AnneJohan Annema, Bram Nauta "Physical and electrical bandwidths of integrated photodiodes in standard CMOS technology", EDSSC2003, 16-18 December HongKong, pp. 95-98. 